\title{
Front Matter: Volume 11823
}

, "Front Matter: Volume 11823," Proc. SPIE 11823, Techniques and Instrumentation for Detection of Exoplanets X, 1182301 (17 September 2021); doi: $10.1117 / 12.2606260$

EDIE Event: SPIE Optical Engineering + Applications, 2021, San Diego, California, United States 


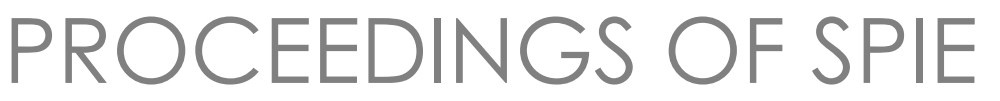

\title{
Techniques and Instrumentation for Detection of Exoplanets $X$
}

\author{
Stuart B. Shaklan \\ Garreth J. Ruane \\ Editors
}

1-5 August 2021

San Diego, California, United States

Sponsored and Published by

SPIE 
The papers in this volume were part of the technical conference cited on the cover and title page. Papers were selected and subject to review by the editors and conference program committee. Some conference presentations may not be available for publication. Additional papers and presentation recordings may be available online in the SPIE Digital Library at SPIEDigitalLibrary.org.

The papers reflect the work and thoughts of the authors and are published herein as submitted. The publisher is not responsible for the validity of the information or for any outcomes resulting from reliance thereon.

Please use the following format to cite material from these proceedings:

Author(s), "Title of Paper," in Techniques and Instrumentation for Detection of Exoplanets X, edited by Stuart B. Shaklan, Garreth J. Ruane, Proc. of SPIE 11823, Seven-digit Article CID Number

(DD/MM/YYYY); (DOI URL).

ISSN: 0277-786X

ISSN: 1996-756X (electronic)

ISBN: 9781510644847

ISBN: 9781510644854 (electronic)

Published by

SPIE

P.O. Box 10, Bellingham, Washington 98227-0010 USA

Telephone +1 3606763290 (Pacific Time)

SPIE.org

Copyright @ 2021 Society of Photo-Optical Instrumentation Engineers (SPIE).

Copying of material in this book for internal or personal use, or for the internal or personal use of specific clients, beyond the fair use provisions granted by the U.S. Copyright Law is authorized by SPIE subject to payment of fees. To obtain permission to use and share articles in this volume, visit Copyright Clearance Center at copyright.com. Other copying for republication, resale, advertising or promotion, or any form of systematic or multiple reproduction of any material in this book is prohibited except with permission in writing from the publisher.

Printed in the United States of America by Curran Associates, Inc., under license from SPIE.

Publication of record for individual papers is online in the SPIE Digital Library.

\section{SPIE. DIGITAL}

Paper Numbering: A unique citation identifier (CID) number is assigned to each article in the Proceedings of SPIE at the time of publication. Utilization of CIDs allows articles to be fully citable as soon as they are published online, and connects the same identifier to all online and print versions of the publication. SPIE uses a seven-digit CID article numbering system structured as follows:

- The first five digits correspond to the SPIE volume number.

- The last two digits indicate publication order within the volume using a Base 36 numbering system employing both numerals and letters. These two-number sets start with 00, 01, 02, 03, 04, 05, 06, 07, 08, 09, 0A, OB ... 0Z, followed by 10-1Z, 20-2Z, etc. The CID Number appears on each page of the manuscript. 


\section{Contents}

\section{GROUND-BASED INSTRUMENTS}

1182302 High resolution spectroscopy of directly imaged exoplanets with KPIC (Invited Paper) [1 1823-1]

1182303 SCEXAO: a testbed for developing high-contrast imaging technologies for ELTs [11823-2]

1182304 A new type of exoplanet direct imaging search: a SCEXAO/CHARIS survey of accelerating stars [1 1823-3]

1182306 Data-driven subspace predictive control: lab demonstration and future outlook [11823-8]

1182308 Information content approach to trade studies for SCALES [1 1823-7]

1182309 The Planetary Systems Imager adaptive optics system: an initial optical design and performance analysis tool for the PSI-Red AO system [11823-9]

IMAGING, SPECTROSCOPY, AND POLARIZATION I

$118230 \mathrm{~A} \quad$ Broadband vortex fiber nulling: high-dispersion exoplanet science at the diffraction limit [11823-12]

11823 OB Design considerations of photonic lanterns for diffraction-limited spectrometry [11823-13]

$118230 \mathrm{C} \quad$ Very high angular resolution spectro-interferometry with wavefront sensing capabilities on Subaru/SCEXAO using photonics [1 1823-11]

11823 OD High-contrast integral field spectropolarimetry of planet-forming disks with SCEXAO/CHARIS [11823-10]

11823 OE From colors to chemistry: a combined lenslet/slicer IFS for medium-resolution spectroscopy [11823-14]

MULTI-PLANET SYSTEMS

11823 OF Planet matching and orbit determination in multi-planet systems for exoplanet direct imaging [11823-15] 
IMAGING, SPECTROSCOPY, AND POLARIZATION II

11823 OG Imaging low-mass planets within the habitable zones of nearby stars with ground-based mid-infrared imaging (Invited Paper) [1 1823-16]

$11823 \mathrm{OH} \quad$ Simulating JWST high contrast observations with PanCAKE [1 1823-17]

$118230 \mathrm{~J}$ Testing a 10 micron HgCdTe detector for ground-based exoplanet science [11823-18]

11823 OK Trade-off study of a high-resolution spectrograph on a CubeSat to study exoplanets [1 1823-20]

DEFORMABLE MIRRORS

$11823 \mathrm{OL} \quad$ Deriving deformable mirror performance requirements in simulation [1 1823-32]

$11823 \mathrm{OM}$ Environmental testing of high-actuator-count MEMS deformable mirrors for space-based applications [11823-33]

\section{CORONAGRAPHS I}

1182300 Results from the laboratory demonstration of a PIAACMC coronagraph with a segmented aperture [1 1823-25]

$11823 \mathrm{OP} \quad$ New method to achieve the proper polarization state for a vector vortex coronagraph [11823-26]

$118230 Q \quad$ High contrast demonstrations of novel scalar vortex coronagraph designs at the high contrast spectroscopy testbed [1 1823-80]

11823 OR Model validation of phase-induced amplitude apodization complex mask coronagraph for LUVOIR-A in vacuum [1 1823-24]

11823 OS High contrast imaging with MEMS deformable mirrors in the Decadal Survey testbed [1 1823-23]

\section{CORONAGRAPHS II}

11823 OV Wavefront control with algorithmic differentiation on the HiCAT testbed [1 1823-28]

$11823 \mathrm{OW}$ Theoretical performance limits for coronagraphs on obstructed and unobstructed apertures: how much can current designs be improved? [11823-31]

11823 OX The PICTURE-C exoplanetary direct imaging balloon mission: first flight preparation [1 1823-30]

iv 
11823 OY The Twin decadal survey testbeds in the high contrast imaging testbed facility at NASA's Jet Propulsion Laboratory [1 1823-22]

\section{ROMAN CORONAGRAPH INSTRUMENT}

1182310 The Roman Space Telescope coronagraph technology demonstration: current status and relevance to future missions (Invited Paper) [1 1823-79]

$1182311 \quad$ Enabling binary stars high-contrast imaging on the Roman Space Telescope coronagraph instrument [1 1823-54]

\section{STARSHADES}

1182312 Optical experiments and model validation of perturbed starshade designs [1 1823-56]

1182313 Catching the rays: modeling the stray light background of a starshade [11823-57]

WAVEFRONT SENSING AND CONTROL I

$1182318 \quad$ High contrast imaging at the photon noise limit with self-calibrating WFS/C systems [1 1823-37]

$118231 \mathrm{~A} \quad$ Wavefront sensing using non-redundant aperture masking interferometry: tests and validation on Subaru/SCEXAO [11823-39]

11823 1B The bright pyramid wavefront sensor [11823-41]

11823 1C Data-driven subspace predictive control: lab demonstration and future outlook [11823-42]

11823 1D The Santa Cruz Extreme AO Lab (SEAL): design and first light [1 1823-35]

$118231 \mathrm{E} \quad$ Fast and furious focal-plane wavefront sensing at W.M. Keck Observatory [11823-36]

11823 IF Status of predictive wavefront control on Keck II adaptive optics bench: on-sky coronagraphic results [1 1823-43]

\section{WAVEFRONT SENSING AND CONTROL II}

$118231 \mathrm{H} \quad$ First experimental results of the fast atmospheric self-coherent camera technique on the Santa Cruz extreme adaptive optics laboratory testbed: demonstration of high speed focal plane wavefront control of residual atmospheric speckles [1 1823-76] 
$118231 \mathrm{~J} \quad$ Strategies for high order wavefront sensing and control (HOWFSC) computation on future space telescopes [11823-47]

$118231 \mathrm{~K}$ Dark zone maintenance results for segmented aperture wavefront error drift in a high contrast space coronagraph [1 1823-48]

11823 IL LUVOIR-ECLIPS closed-loop adaptive optics performance and contrast predictions [11823-50]

$118231 \mathrm{M}$ Experimental validation of active control of low-order aberrations with a Zernike sensor through a Lyot coronagraph [1 1823-46]

1182310 Multi-star wavefront control with SCExAO instrument: demonstration with an internal source [11823-51]

$118231 \mathrm{P} \quad$ Initial super-Nyquist wavefront control experiments in the Decadal Survey Testbed [1 1823-52]

POSTER SESSION

$118231 Q$ Implementation of a broadband focal plane estimator for high-contrast dark zones [1 1823-62]

11823 IR Performance of large-format deformable mirrors constructed with hybrid variable reluctance actuators II: initial lab results from FLASH [1 1823-69]

11823 is Design and commissioning of an in-air coronagraph testbed in the HCIT facility at NASA's Jet Propulsion Laboratory [11823-78]

$118231 \mathrm{~T} \quad$ Dual-polarization electric field conjugation and applications for vector vortex coronagraphs [11823-64]

$118231 \mathrm{U}$ Confirming transiting exoplanets with the Fraunhofer Telescope Wendelstein [1 1823-63]

11823 IV Cold-stop and Lyot stop designs for a new Infrared Exoplanet Imager at Keck Observatory [11823-66]

11823 IW Cryogenic test results of the SCALES focal plane coronagraph mechanism [1 1823-68]

$118231 Y \quad$ Flight mask designs of the Roman Space Telescope coronagraph instrument [1 1823-72] 